\title{
Supertransport of excitons in atomically thin organic semiconductors at the 2D quantum limit
}

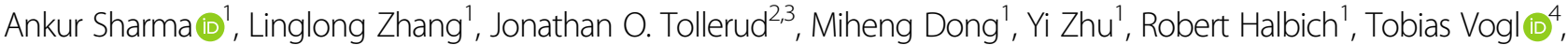 \\ Kun Liang ${ }^{1,5}$, Hieu T. Nguyen (1) ${ }^{1}$, Fan Wang ${ }^{6}$, Shilpa Sanwlani ${ }^{2,3}$, Stuart K. Earl $\mathbb{B}^{2,3}$, Daniel Macdonald ${ }^{1}$, Ping Koy Lam (1) ${ }^{4}$, \\ Jeffrey A. Davis $\mathbb{1}^{2,3}$ and Yuerui Lu ${ }^{1,3,4}$
}

\begin{abstract}
Long-range and fast transport of coherent excitons is important for the development of high-speed excitonic circuits and quantum computing applications. However, most of these coherent excitons have only been observed in some low-dimensional semiconductors when coupled with cavities, as there are large inhomogeneous broadening and dephasing effects on the transport of excitons in their native states in materials. Here, by confining coherent excitons at the 2D quantum limit, we first observed molecular aggregation-enabled 'supertransport' of excitons in atomically thin two-dimensional (2D) organic semiconductors between coherent states, with a measured high effective exciton diffusion coefficient of $\sim 346.9 \mathrm{~cm}^{2} / \mathrm{s}$ at room temperature. This value is one to several orders of magnitude higher than the values reported for other organic molecular aggregates and low-dimensional inorganic materials. Without coupling to any optical cavities, the monolayer pentacene sample, a very clean 2D quantum system $(\sim 1.2 \mathrm{~nm}$ thick $)$ with high crystallinity (J-type aggregation) and minimal interfacial states, showed superradiant emission from Frenkel excitons, which was experimentally confirmed by the temperature-dependent photoluminescence (PL) emission, highly enhanced radiative decay rate, significantly narrowed PL peak width and strongly directional in-plane emission. The coherence in monolayer pentacene samples was observed to be delocalised over 135 molecules, which is significantly larger than the values (a few molecules) observed for other organic thin films. In addition, the supertransport of excitons in monolayer pentacene samples showed highly anisotropic behaviour. Our results pave the way for the development of future high-speed excitonic circuits, fast OLEDs, and other optoelectronic devices.
\end{abstract}

\section{Introduction}

Recently, there has been increasing interest in harnessing the long-range and fast transport of coherent excitons (electron-hole pair quasi-particles) in solid-state inorganic semiconductors and molecular systems with confined geometries to enhance light-matter interactions ${ }^{1-3}$. Coherence is critical to engineering the quantum electrodynamics $(\mathrm{QED})^{4}$ in low-dimensional systems, such as quantum wells ${ }^{5}$, two-dimensional (2D) materials ${ }^{6}$,

\footnotetext{
Correspondence: Yuerui Lu (yuerui.lu@anu.edu.au)

${ }^{1}$ Research School of Electrical, Energy and Materials Engineering, College of Engineering and Computer Science, The Australian National University, Canberra, ACT 2601, Australia

${ }^{2}$ Optical Sciences Centre, Swinburne University of Technology, Hawthorn, VIC 3122, Australia

Full list of author information is available at the end of the article
}

and quantum $\operatorname{dots}^{7}$, leading to various applications in realising quantum memories ${ }^{8}$, single-photon sources ${ }^{9}$, laser cooling ${ }^{10}$, narrow linewidth lasers ${ }^{11}$, high efficiency solar cells ${ }^{12}$, and stable polaritons to achieve Bose Einstein condensates ${ }^{5}$. Long-range and fast migration of coherent excitons also has tremendous applications in schemes of high-speed excitonic circuits, quantum computing ${ }^{13-15}$, and high quantum yield light-emitting diodes ${ }^{16}$.

Spontaneous coherent emission from a system of several non-interacting dipole active atoms (excitons) was defined as superradiance (SR) by Dicke ${ }^{17}$. In this phenomenon, interactions between transition dipoles of individual molecules allow coherent delocalisation across multiple sites. This leads to a net enhancement in the optical transition dipole moment (TDM) and a sharp

\section{(c) The Author(s) 2020}

\footnotetext{
(c) Open Access This article is licensed under a Creative Commons Attribution 4.0 International License, which permits use, sharing, adaptation, distribution and reproduction in any medium or format, as long as you give appropriate credit to the original author(s) and the source, provide a link to the Creative Commons license, and indicate if changes were made. The images or other third party material in this article are included in the article's Creative Commons license, unless indicated otherwise in a credit line to the material. If material is not included in the article's Creative Commons license and your intended use is not permitted by statutory regulation or exceeds the permitted use, you will need to obtain permission directly from the copyright holder. To view a copy of this license, visit http://creativecommons.org/licenses/by/4.0/.
} 
enhancement of the excitonic radiative decay rate of an ensemble of $N_{c}$ independent emitters compared to the radiative decay rate of a single emitter ${ }^{2,7}$. The same principle of coherent delocalised superradiant emission gives rise to an analogous phenomenon called cooperative energy transfer or supertransfer $(\mathrm{ST})^{18}$. The resulting enhanced oscillator strength from delocalisation over large molecular assemblies can lead to large-scale exciton transport. In an ST process, the molecular assemblies (consisting of $N_{c}$ molecules) with comparable net TDMs can play the role of acceptors similar to individual molecules in the Förster resonance energy transfer (FRET) mechanism ${ }^{19}$. Thus, the excitation (exciton) can be transferred over much longer distances in a delocalised molecular assembly before annihilation, resulting in large effective exciton diffusion coefficients.

Recently, coherent exciton transport with highly enhanced effective exciton diffusion coefficients $\left(3-70 \mathrm{~cm}^{2} / \mathrm{s}\right)^{20-22}$ was observed in quasi-one-dimensional (quasi-1D) molecular assemblies (cylindrical bundles ${ }^{20}$, nanotubes $^{19}$ and wires ${ }^{21,22}$ ), and these coefficients were significantly higher than the migration speeds of incoherent excitons reported in other materials, including conventional organic thin films and their heterostructures $\left(0.001-3 \mathrm{~cm}^{2} / \mathrm{s}\right)^{23,24}$, III-V semiconductor quantum wells $\left(0.1-10 \mathrm{~cm}^{2} / \mathrm{s}\right)^{25}$, atomically thin transition metal dichalcogenides (TMDs) and phosphorene $\left(0.01-14.5 \mathrm{~cm}^{2} / \mathrm{s}\right)^{26}$, etc. (Table S1 in the Supplementary Information). However, the migration speeds of the coherent excitons in these quasi-1D systems ${ }^{20-22}$ were still hindered by the low overall exciton oscillation strengths (low coherence length with small $N_{\mathrm{c}}$ values), which were mainly limited by the low quantum confinement of excitons ${ }^{27}$ (diameter size $>12 \mathrm{~nm}$ ) and potential disorder and interfacial sites in the systems $^{28}$. Additionally, these quasi-1D systems had very small cross-section areas for light-matter interactions, limiting their applications in future excitonic devices ${ }^{3,29-31}$. As predicted by theory, the exciton oscillation strength, a key parameter for coherent excitons, is very sensitive to the quantum confinement of the system ${ }^{27}$. Therefore, confining coherent excitons at the 2D quantum limit is a very promising way to realise long-range and fast transport of excitons; moreover, the 2D structure can also provide a large cross-section area for light-matter interactions, enabling tremendous applications in future excitonic devices.

In this work, in atomically thin organic molecular crystals without any optical cavities, we observe longrange and fast migration of Frenkel (FR) excitons between coherent states at the $2 \mathrm{D}$ quantum limit. A very high effective exciton diffusion coefficient of $\sim 346.9 \mathrm{~cm}^{2} / \mathrm{s}$ at room temperature is reported, which is one order of magnitude higher than the values previously reported (Table S1 in the Supplementary Information) for other materials ${ }^{20,22,32}$. The sharp, strong emission arising from J-type (monolayer, shortened as $1 \mathrm{~L}$ ) aggregation in pentacene in contrast to the emission arising from $\mathrm{H}$-type (wetting layer, shortened as WL) aggregation is confirmed to be superradiant emission from FR excitons. The coherence in $1 \mathrm{~L}$ pentacene samples is determined to be delocalised over $\sim 135$ molecules, which is more than one order of magnitude larger than the values (a few molecules) observed in other organic thin films ${ }^{33}$. Our simulation results from quantum calculations attribute this to the constructive dipole coupling in the J-aggregates in $1 \mathrm{~L}$ pentacene that forms an enhanced net optical dipole moment, which supports our experimental observations. The supertransport of excitons observed on the macroscopic scale will enable realisation of strongly enhanced light-matter interactions at the quantum limit using such 2D organic materials, which has key applications in developing high-speed quantum computing devices, fast response time OLEDs, excitonic transistors and other optoelectronic devices ${ }^{8,15,31}$.

\section{Results \\ Sample growth and characterization}

Single-crystal pentacene (Fig. 1) was epitaxially grown layer by layer on a hexagonal boron nitride (h-BN) surface with atomic smoothness and well-defined crystal facets. The first pentacene layer on $\mathrm{h}-\mathrm{BN}$ was named the wetting layer (WL; $\sim 0.6 \mathrm{~nm}$ thick), and the next layer of pentacene grown on WL was designated the monolayer $(1 \mathrm{~L} ; 1.2 \mathrm{~nm}$ thick $)^{34}$ (Fig. 1a-c). The layer-dependent arrangement of pentacene molecules over $\mathrm{h}-\mathrm{BN}$ and their electrical transport properties were described in a recent report ${ }^{34}$. Raman spectroscopy was used to confirm the presence of pentacene on h-BN (Fig. S1). PL spectroscopy with a continuous-wave $532 \mathrm{~nm}$ excitation laser was used to characterise the excitonic emissions from the atomically thin pentacene layers. The WL region showed much stronger PL emissions than the $1 \mathrm{~L}$ region at room temperature (Figs. 1d and S2 and associated text). Compared with inorganic TMD 2D semiconductors, WL showed a much broader PL spectrum at room temperature, which is associated with the various band energy levels formed in pentacene due to vibronic couplings between FR and charge-transfer (CT) excitons (Supplementary Information note 1 and Fig. S3) ${ }^{35,36}$. On the other hand, the $1 \mathrm{~L}$ region showed a single peak centred at $\sim 680 \mathrm{~nm}$ that corresponds predominantly to FR excitonic emission, which will be explained later. This region is a heterostructure consisting of $1 \mathrm{~L}$ on top of WL. However, in the PL spectrum from the $1 \mathrm{~L}$ region, we did not see the higher-energy emission $(\sim 550-650 \mathrm{~nm})$ observed in the WL sample (Fig. 1d) because the photoexcited charges in the WL underneath could be quickly transferred to $1 \mathrm{~L}$ before radiative emission occurred (Fig. S2). Therefore, 

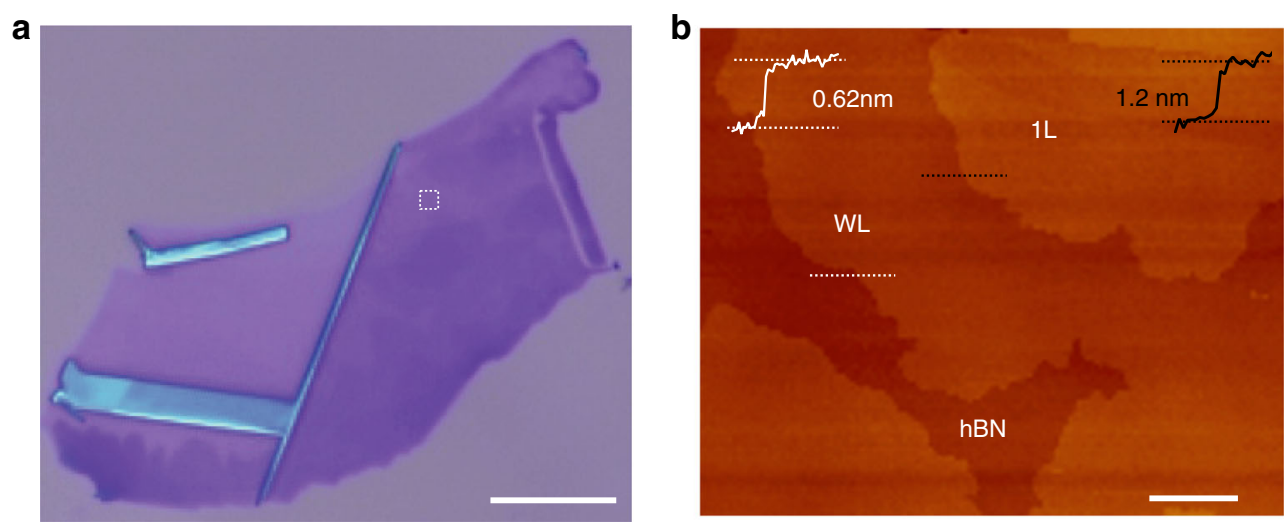

C

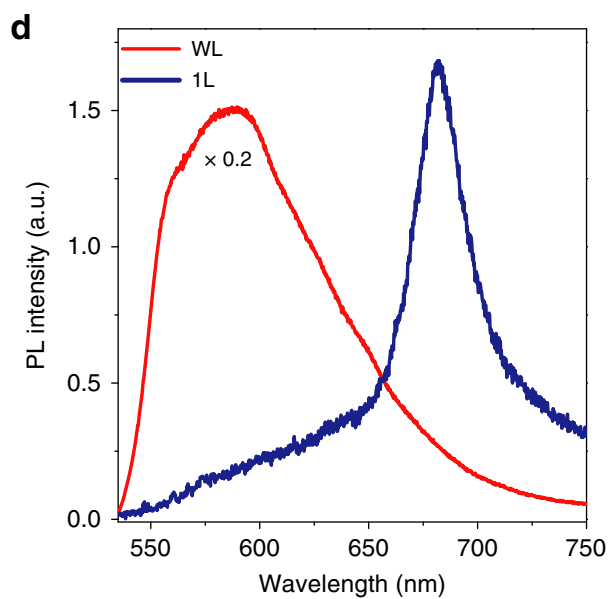

Fig. 1 Characterization of atomically thin 2D layered pentacene samples. a Optical microscope image of the sample used for measurements. The scale is $80 \mu \mathrm{m}$. b Zoomed-in atomic force microscopy (AFM) image of the dashed square region in $\mathbf{a}$, showing the actual measured thicknesses of $\mathrm{WL}$ and $1 \mathrm{~L}$ pentacene. The scale is $2 \mu \mathrm{m}$. c Schematic diagram showing the orientation and alignment of pentacene molecules in WL and $1 \mathrm{~L}$ over h-BN and a $\mathrm{SiO}_{2} / \mathrm{Si}$ substrate. $\mathbf{d}$ Measured PL spectra from WL and $1 \mathrm{~L}$ samples at room temperature

the PL spectrum measured from the $1 \mathrm{~L}$ region comes from the $1 \mathrm{~L}$ pentacene sample and reflects its intrinsic nature.

\section{Superradiance and excitonic characteristics}

To explore the nature of excitonic transitions, we conducted temperature-dependent PL measurements on the WL and 1L pentacene samples down to $77 \mathrm{~K}$ (Fig. 2). With decreasing temperature, the PL intensity from WL dropped significantly, and the full-width at half-maximum (FWHM) broadened (Fig. 2a); in contrast, 1L pentacene showed a sharp rise in the PL intensity and a significantly reduced FWHM with decreasing temperature (Figs. 2b and S4). The opposing temperature dependences were caused by the completely different molecular packings in WL and 1L pentacene, which can be fully understood based on the theory of $\mathrm{H}$ - and J-type molecular aggregation and their effects on optical emission ${ }^{35,37}$ (Supplementary Information note 2; Fig. S3). Theoretical values of the coupling coefficients $(J)$ and net TDM in each layer were extracted based on the molecular aggregation pattern and coupling (Fig. S3a). WL exhibited a parallel packing of TDMs, and $1 \mathrm{~L}$ exhibited a head-to-tail packing of TDMs because of the herringbone packing of pentacene molecules in the unit cell (Figs. 1c and S3) ${ }^{38}$. The $J$ values for $1 \mathrm{~L}$ and $\mathrm{WL}$ pentacene were negative and positive, respectively, suggesting $\mathrm{J}$ - and $\mathrm{H}$-type aggregation for $1 \mathrm{~L}$ and $\mathrm{WL}$, respectively ${ }^{38}$. The total TDM and oscillator strength for the lowest excited states $\left(S_{1}\right)$ in WL were both zero, leading to non-luminescent $S_{1}$ states at a temperature of absolute zero. In contrast, the total TDM for $S_{1}$ in $1 \mathrm{~L}$ had a large value (with the direction along the $b$ axis of the unit cell), and its corresponding oscillator strength was high (Fig. S3a). This resulted in luminescent $S_{1}$ states at absolute zero. The large magnitude of the net TDM corresponds to phase locking and constructive coupling of TDMs in $1 \mathrm{~L}$ pentacene cooperatively, resulting in a sharp rise in the PL intensity and a reduction in the FWHM, which are characteristics of superradiant emission $^{33,35,39}$. 


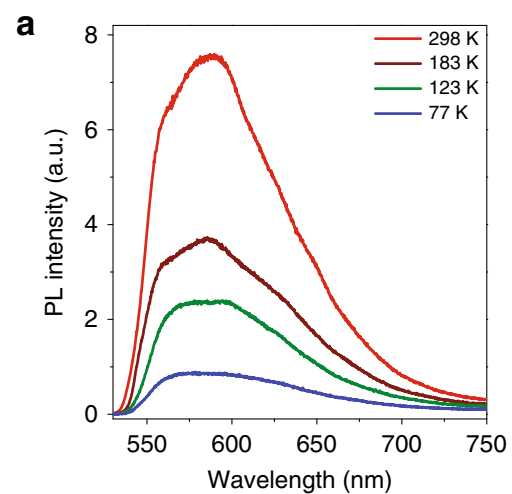

b

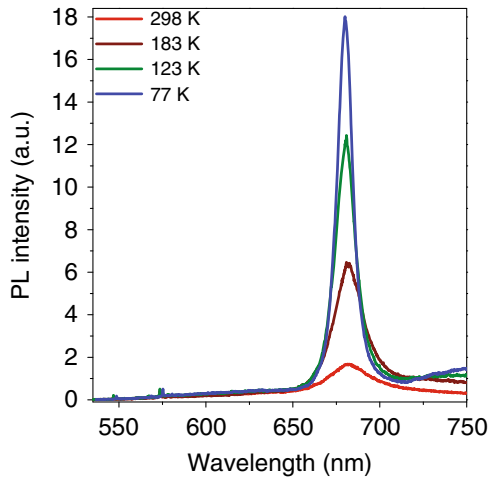

C

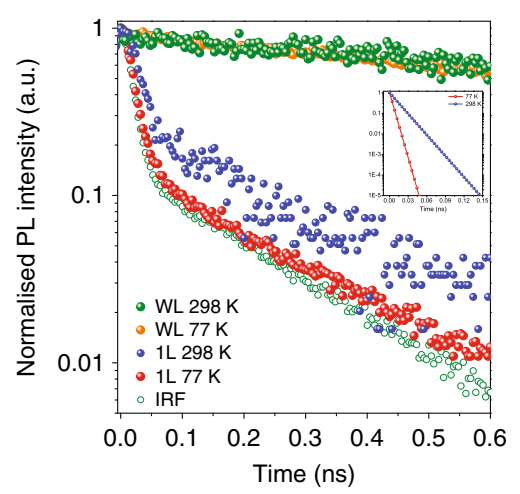

d
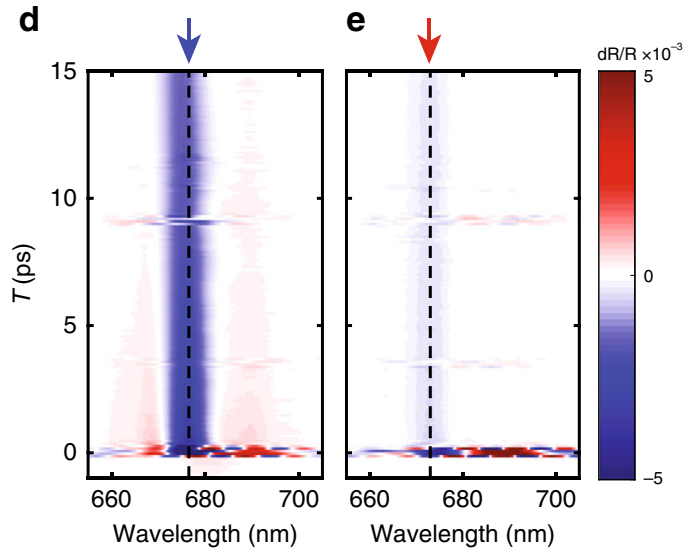

f

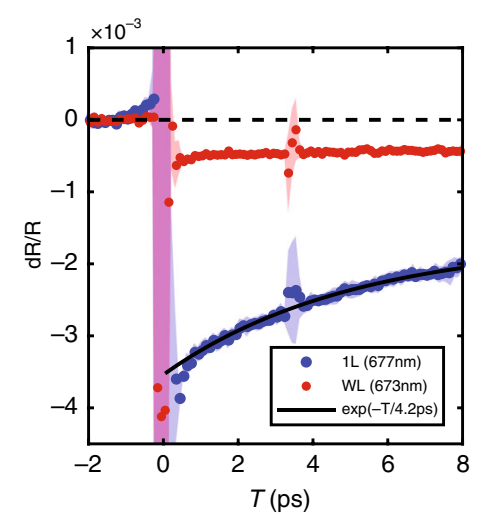

g

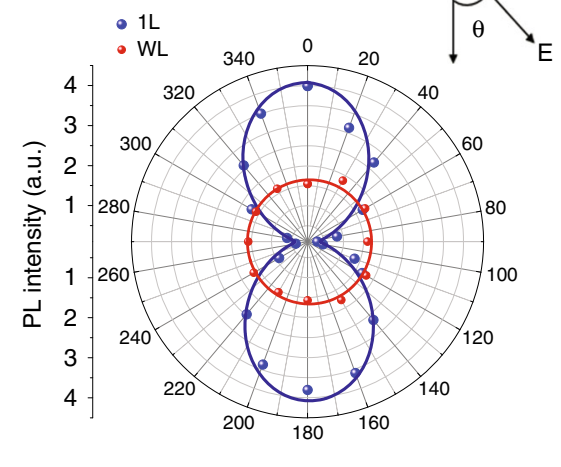

Fig. 2 Temperature-dependent optical measurements. a, b PL spectra measured at various temperatures for WL (a) and $1 \mathrm{~L}$ (b). For $1 \mathrm{~L}$, a sharp peak at $\sim 680 \mathrm{~nm}$ (designated as FR excitonic emission) rises sharply as the temperature decreases. c Time-resolved PL emission (normalised) from WL and $1 \mathrm{~L}$ pentacene samples at 77 and $298 \mathrm{~K}$. The orange/green balls represent the measured decay curve from WL, which arises from CT exciton emission. An effective long lifetime of $2.64 \mathrm{~ns}(2.89 \mathrm{~ns})$ was extracted from the orange (green) decay curve at $77 \mathrm{~K}$ ( $298 \mathrm{~K}$ ) by fitting with deconvolution of the instrument response function (IRF) (green dots). The red/blue balls represent the decay curve from 1L, which arises from FR exciton emission. The red (blue) decay curve was fitted (solid line) with deconvolution of the IRF, giving an effective short lifetime of 4.1 ps (12.7 ps) for $1 \mathrm{~L}$ at $77 \mathrm{~K}(298 \mathrm{~K})$. The inset shows the deconvoluted curves obtained from $1 \mathrm{~L}$. d, e Time- and spectrally resolved transient differential reflectance of $1 \mathrm{~L}$ (c) and WL (d) recorded using $200 \mathrm{fs}$ degenerate pump and probe with spectra centred at $680 \mathrm{~nm}$. f Slices of the TR for $1 \mathrm{~L}$ (blue) and WL (red) at $677 \mathrm{~nm}$ and $673 \mathrm{~nm}$, respectively. A lifetime of $4.2 \mathrm{ps}$ was extracted from the $1 \mathrm{~L}$ decay, while the WL response was effectively constant across the range. The feature at $3.5 \mathrm{ps}$ is due to interference between the pump pulse and a weak reflection of the probe pulse. $\mathbf{g}$ Measured polar plot of PL intensity as a function of emission polarisation angle $\theta$ from $1 \mathrm{~L}$ (blue) and $\mathrm{WL}$ (red) pentacene samples at $77 \mathrm{~K}$, revealing the anisotropic (isotropic) excitonic nature of emission from $1 \mathrm{~L}(\mathrm{WL})$ pentacene. In the experiment, the excitation polarisation angle was fixed, and the polarisation angle of the emission $\theta$ was determined by an angle-variable polarizer located in front of the detector. The solid lines are curves fitted using a $\cos ^{2} \theta$ function 
The phenomenon of superradiance is the combined process of coherent emission from a cluster of $N_{\mathrm{c}}$ molecular aggregates/chromophores whose oscillator strength is enhanced compared to that of a single emitter by a factor of $N_{\mathrm{c}}$ owing to the large TDM associated with the molecular assembly ${ }^{33,37}$. To determine which coherent size gives the best agreement with the experimental temperature dependence of the PL intensity, we used the Huang-Rhys factor and the spectral strengths of the 0-0 and $0-1$ transitions in the 1L PL spectrum to accurately determine the coherence number and hence the effect of vibrations and phononic interactions on the coherence length ${ }^{36}$. The $N_{\mathrm{c}}$ value can also be estimated by the sharp reduction in the FWHM and spectral strengths of the PL emission as the temperature decreases ${ }^{39}$. Based on this technique and our measurements in Figs. 2c, S4 and S5, we estimated this value to be over 135 molecules. (See Supplementary Information note 3.) The extracted $N_{\mathrm{c}}$ value for our $1 \mathrm{~L}$ pentacene sample was more than one order of magnitude larger than that observed for tetracene thin film $\left(N_{\mathrm{c}} \sim\right.$ a few molecules) ${ }^{33}$. The $N_{\mathrm{c}}$ value signifies the extent of FR exciton delocalisation, which was over 135 consecutive dipoles in $1 \mathrm{~L}$ pentacene. The enhanced oscillator strength was distributed over these consecutive dipoles, resulting in a large net TDM, as shown in Fig. S3.

Excitonic superradiance also leads to shortening of the excitonic radiative decay lifetime for the emis$\operatorname{sion}^{2,4,7,11,33,39}$ by a factor of $N_{c}$. To confirm this coherent superradiance from $1 \mathrm{~L}$, we measured the temperaturedependent lifetimes of two excitonic emissions from WL and $1 \mathrm{~L}$ pentacene samples using time-resolved photoluminescence (TRPL) measurements with a resolution of $2.1 \mathrm{ps}$ after deconvolution (Fig. S6). The lifetime observed for the $1 \mathrm{~L}$ pentacene sample significantly dropped from $\sim 12.7 \pm 0.8 \mathrm{ps}$ at room temperature to $\sim 4.1 \pm 0.7 \mathrm{ps}$ at $77 \mathrm{~K}$ (Fig. 2c). To further confirm the measurement accuracy of the lifetime, we performed time- and spectrally resolved differential transient reflectance (TR) measurements on WL and $1 \mathrm{~L}$ pentacene samples at $77 \mathrm{~K}$ using a pump-probe technique with a time resolution of $200 \mathrm{fs}$. The $1 \mathrm{~L}$ pentacene sample showed a clear dip in the differential TR mapping centred at $677 \mathrm{~nm}$; in contrast, the dip was not clear for the WL sample (Fig. 2d, e). The relaxation of this feature in the $1 \mathrm{~L}$ sample followed a fast decay (lifetime of $\sim 4.2 \pm 0.53 \mathrm{ps;}$ Fig. 2f). Fitting of the decay curve was performed using a single exponential decay and a $95 \%$ confidence interval, giving an estimated lifetime range of 3.6-4.7 ps (Fig. S7). Further details about the measurements and the fitting are explained in Supplementary Information Fig. S7 and the associated text. The consistency between the two complementary techniques confirms the accuracy of our lifetime values measured with our TRPL system. The significant reduction in the emission lifetime with decreasing temperature is consistent with the observed sharp rise in the PL intensity in the $1 \mathrm{~L}$ pentacene sample (Fig. $2 \mathrm{~b}$ ), confirming the fast and radiative nature of the decay, as expected for superradiance.

Furthermore, the radiative lifetime of singlet FR excitonic emissions in bulk pentacene thin films (which are non-coherent monomer states) was experimentally established to be $\sim 1200 \mathrm{ps}^{40}$. We also confirmed the lifetime in our bulk thin film pentacene grown on the same h-BN substrate, which was measured to be $1241.1 \mathrm{ps}$ at room temperature (Fig. S8 and associated text). The significantly reduced lifetime $(\sim 12.7 \mathrm{ps})$ in our $1 \mathrm{~L}$ pentacene sample compared to the value $(\sim 1200 \mathrm{ps})$ for the non-coherent states in thin film pentacene, coupled with the consistent temperature-dependent PL emission and lifetime, further substantiates superradiant emission from $1 \mathrm{~L}$ pentacene. The value of $N_{\mathrm{c}}$, as extracted from this sharp reduction in lifetime, was $\sim 100$ at room temperature, which is again in close agreement with the aforementioned extracted values based on the highly enhanced PL intensity and reduced FWHM with decreasing temperature. In contrast, the lifetimes for the WL pentacene sample were measured to be $2.89 \mathrm{~ns}$ using TRPL at room temperature and $2.64 \mathrm{~ns}$ at $77 \mathrm{~K}$ (Fig. 2c), much larger than the values from the $1 \mathrm{~L}$ pentacene samples, confirming the non-coherent nature of the CT excitons in the WL samples.

We further performed PL measurements with angleresolved excitation and emission polarisation (Figs. $2 \mathrm{~g}$ and S9) to confirm the anisotropic nature of the superradiant FR excitonic emissions from 1L. The PL intensity from $1 \mathrm{~L}$ strongly depended on the emission polarisation angle $\theta$ and showed a period of $180^{\circ}$; in contrast, the emission from WL was isotropic (Fig. $2 \mathrm{~g}$ ). The theoretically predicted strongly directional total TDM in $1 \mathrm{~L}$ pentacene (Fig. S3a) is expected to lead to an in-plane anisotropic emission of the FR excitons. Henceforth, the zero-degree reference in the polar plots (Fig. 2f) and subsequent figures can be identified as the $b$ axis of the unit cell in 1L pentacene (Figs. 1c and S3a). The directional emission in the $1 \mathrm{~L}$ pentacene sample was independent of the excitation polarisation angle of the incident laser (Fig. S9). A similar anisotropy for the PL emission from $1 \mathrm{~L}$ pentacene was observed for $1 \mathrm{~L}$ samples at room temperature (Fig. $\mathrm{S} 10)$. Normally, the dichroic ratio $(D R)$ is a term used to characterise the in-plane anisotropy of optical transitions ${ }^{41}$. Here, it is defined as $\mathrm{DR}=\mathrm{I}_{\|} / \mathrm{I}_{\perp}$, where $\mathrm{I}_{\|}$and $\mathrm{I}_{\perp}$ are the PL peak intensities measured at polarisation angles of $0^{\circ}$ and $90^{\circ}$, respectively. The measured DR values of PL emission from $1 \mathrm{~L}$ for emission (Fig. 2g, blue curve) polarisation at $77 \mathrm{~K}$ were high, up to 10.3 , which indicates the strongly anisotropic nature of the PL emission from $1 \mathrm{~L}$. At room temperature, the DR value observed for $1 \mathrm{~L}$ samples was close to 5.9. In sharp contrast, the DR values 
measured from the PL emission in WL pentacene were isotropic both at room temperature and $77 \mathrm{~K}$ (Fig. 2g, red curve), which suggests that these peaks are dominated by the contribution of CT excitons ${ }^{42}$. Supplementary Information note 4 presents a further detailed discussion on the different natures of the excitonic states in WL and $1 \mathrm{~L}$.

\section{Long-range and superfast exciton transport}

The same principle of coherent delocalised superradiant emission gives rise to an analogous phenomenon called cooperative energy transfer or supertransfer ${ }^{18}$. The enhanced oscillator strength resulting from delocalisation over large molecular assemblies can lead to large-scale exciton transport. The near-field direct imaging technique with a charge-coupled device (CCD) camera ${ }^{21,22,43}$ was used to detect the diffusion length of bright excitons from $\mathrm{WL}$ and $1 \mathrm{~L}$ pentacene samples (Fig. 3). Figure 3a, b shows the measured contour plots of the PL intensity as a function of the emission wavelength and space of exciton diffusion for the $1 \mathrm{~L}(\mathrm{WL})$ pentacene sample at $77 \mathrm{~K}$. The spatial distribution of the PL intensity can be extracted at specific energies (shown by dotted lines in Fig. 3a, b). Figure 3c shows the extent of spatial diffusion in a steady 1D diffusion model. A Gaussian fitting routine ${ }^{21,43}$ was used to quantitatively compare the reflected excitation beam to the emission profile emerging from pentacene. Both the emission and laser profiles were fitted using a bivariate normal distribution, and their FWHMs were extracted. The spatial extent of exciton diffusion (diffusion length $L_{D}$ ) was extracted by taking the difference between the standard deviations of the emission image and the passive laser spot after fitting the raw data. A Gaussian fitting model (see methods) was used because the excitation from the laser profile was predominantly Gaussian and the diffusion model was not pair mediated or defect assisted in our high crystalline sample ${ }^{44,45}$. The exciton diffusion lengths $\left(L_{D}\right)$ in the $1 \mathrm{~L}$ and WL pentacene samples were measured to be $0.55 \pm 0.06 \mu \mathrm{m}$ and $1.36 \pm 0.16 \mu \mathrm{m}$, respectively (Fig. 3d). All the measurements were taken at a low excitation power of $\sim 6 \mu \mathrm{W}$. We also performed measurements with different powers ranging from 6 to $215 \mu \mathrm{W}$, and similar diffusion lengths and lifetimes were observed, which shows the minimal and ignorable effect of exciton-exciton annihilation in our measurements (Fig. S11) ${ }^{19,46}$. Using the measured excitonic emission lifetimes $(\tau)$, the effective exciton diffusion coefficient $\left(D_{\text {eff }}\right)$ could be extracted using the equation $^{22,46} L_{D}=\sqrt{2 D_{\text {eff }} \tau}$. Using the lifetimes (Fig. 2e), the extracted diffusion coefficients for the $1 \mathrm{~L}$ and WL pentacene samples at $77 \mathrm{~K}$ were $354.5 \pm 50.1 \mathrm{~cm}^{2} / \mathrm{s}$ and $3.5 \pm$ $0.2 \mathrm{~cm}^{2} / \mathrm{s}$, respectively (Fig. $3 \mathrm{~d}$ ). The $D_{\text {eff }}$ value obtained from $1 \mathrm{~L}$ is almost one order of magnitude higher than the values reported for other organic/inorganic systems with coherent and delocalised excitonic emissions $\left(3-70 \mathrm{~cm}^{2} /\right.$ sec, Table 1 in Supplementary Information) ${ }^{20-22}$. At the same time, this value is $\sim 3$ orders of magnitude higher than the diffusion constant reported for non-coherent singlet excitons in bulk thin film pentacene by Marciniak et $\mathrm{al}^{40}$. The exciton diffusion coefficients in $\mathrm{WL}$ and $1 \mathrm{~L}$ at $77 \mathrm{~K}$ were further corroborated by another alternative method, spatial-temporal mapping ${ }^{47}$, which is a very robust method for accurately determining the exciton diffusion coefficient in similar materials ${ }^{47}$. Figure $4 \mathrm{a}$ shows the experimental setup used for spatial-temporal mapping, and the obtained data plots stitched together are shown in Fig. 4b, c. (See Methods section) The diffusion coefficient extracted from the spatial-temporal mapping was obtained by extracting the Gaussian dispersion of the emission profile over time and was consistent with our reported values obtained from the time-resolved PL and diffusion mapping in space technique, highlighted in Fig. 3. The diffusion coefficients for $1 \mathrm{~L}$ and $\mathrm{WL}$ were $306.8 \pm$ $14.1 \mathrm{~cm}^{2} / \mathrm{s}$ and $3.3 \pm 1.1 \mathrm{~cm}^{2} / \mathrm{s}$, respectively, as obtained from the slope in Fig. 4d, e. It is important to remark here that the initial diffusion value for WL was non-zero due to cooling of hot $\mathrm{CT}$ excitons in the $<4$ ps regime ${ }^{48}$. As discussed earlier in this report, WL is predominantly CT exciton dominated. For CT excitons in the initial few hundred femtoseconds to few ps regime, the CT excitons undergo cooling or lose excess energy due to the creation of many intermediate energy states between $S_{1}$ (natural excited exciton energy state) and CT states, and they are all electronic states that excitons can quickly jump to in a few $\mathrm{ps}^{24,48}$. Furthermore, due to excitons residing on two different molecules, the extent of movement is also enhanced. All of this occurs in a few hundred femtoseconds to a few ps; hence, the non-zero value in WL can be attributed to hot CT exciton states cooling rapidly to intermediate energy levels created due to H-type aggregation and donor/acceptor-mediated hopping of excitons. Once the initial cooling occurs, the excitons then stabilise and diffuse normally. Since this non-zero transport occurred within the few ps regime, it did not affect the accuracy of our measurement of the exciton diffusion coefficient in WL, where the diffusion stretched to the few hundred ps-ns regime. In $1 \mathrm{~L}$ samples, which are predominantly FR exciton dominated, we did not observe such non-zero transport at $t=0$, as the hot CT excitons were practically non-existent there. The results further confirm the effectiveness of our technique in measuring precise exciton diffusion with a high resolution and the limited role of triplet fission in our samples.

We attribute the high value of the exciton diffusion coefficient in $1 \mathrm{~L}$ to the supertransfer phenomenon. The reason we were able to achieve these large effective exciton diffusion coefficients is threefold. First, in our $1 \mathrm{~L}$ pentacene samples, the strong intermolecular coupling (Jtype aggregation) results in a strong net exciton oscillator 

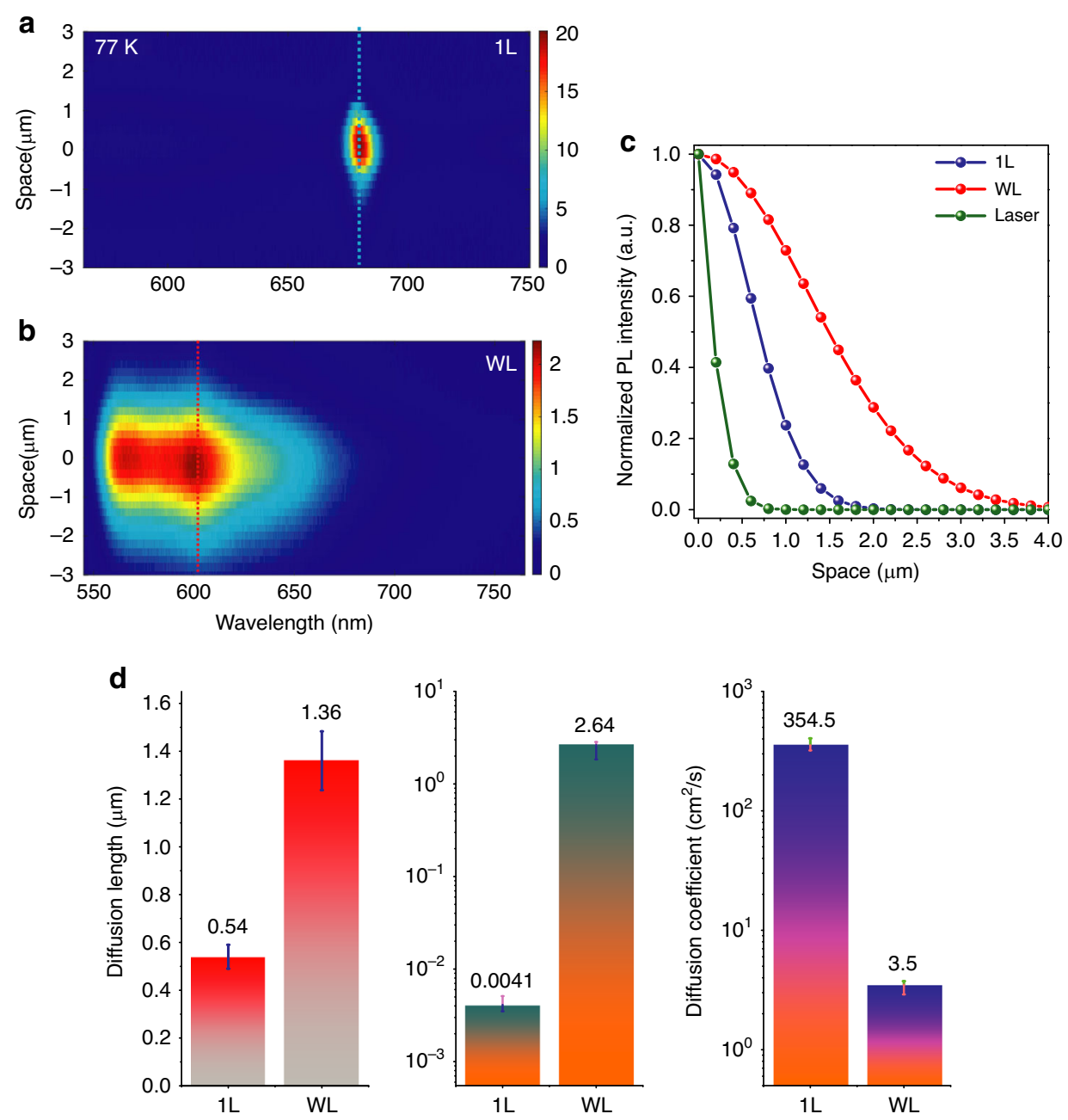

Fig. 3 Exciton transport mapping using near-field imaging. $\mathbf{a}$, b Measured contour plots of PL intensity as a function of emission wavelength and space of exciton diffusion for $1 \mathrm{~L}(\mathbf{a})$ and $\mathrm{WL}(\mathbf{b})$ pentacene samples at $77 \mathrm{~K}$. The middle of the laser excitation spot is at $x=0$. $\mathbf{c}$ Spatial profiles plotted along the dotted lines in $\mathbf{a}$, $\mathbf{b}$ for different types of excitonic peaks, showing the diffusion lengths of excitons in WL and $1 \mathrm{~L}$. $\mathbf{d}$ Comparison plots of diffusion lengths (left; from Fig. 3a-c), lifetime (middle; from Fig. 2e) and extracted diffusion coefficients measured from WL and 1L samples at 77K. The error bars represent the experimental variation observed in measurements of multiple samples

strength and thus delocalised and superradiant excitonic emissions. Within each delocalised segment, the excitation energy propagates ballistically ${ }^{18}$, in contrast to the diffusive-hopping-described FRET. Delocalised excitons in principle accelerate energy transfer compared to incoherent molecular hopping transfer because delocalisation can define an effective hopping length that is much larger than the nearest intermolecular spacings (Supplementary Information note 5) ${ }^{49-51}$. Second, because of the high-quality epitaxial 2D growth, our pentacene samples have highly ordered crystallinity and minimised defects/ disorder, resulting in long delocalisation lengths and large coherence lengths $\left(N_{\mathrm{c}} \sim 135\right.$, as shown in Fig. S5, extracted from temperature-dependent PL line strength measurements), which significantly benefit the transport speed of coherent excitons. The exciton delocalisation length is defined by competition between intermolecular coupling and disorder ${ }^{19}$, which can be clearly seen from the comparison between the PL spectra from high-quality and low-quality samples. In some $1 \mathrm{~L}$ samples with lowquality growth, the FR exciton peak at $\sim 680 \mathrm{~nm}$ was weak; instead, high-energy CT peaks appeared in the PL spectra (Fig. S12; Supplementary Information note 6). Compared with the high-quality $1 \mathrm{~L}$ pentacene samples, as shown in Fig. 2, these low-quality $1 \mathrm{~L}$ samples showed a broader peak and a lower PL peak intensity at $680 \mathrm{~nm}$ and longer radiative lifetimes of the FR excitons (Fig. S13), which was caused by the reduced coherence length in the low-quality $1 \mathrm{~L}$ samples. In the experiment, we also found that the disorder and interfacial states increased as the sample thickness increased, which led to decoherence and reduced exciton delocalisation length in thick samples, 

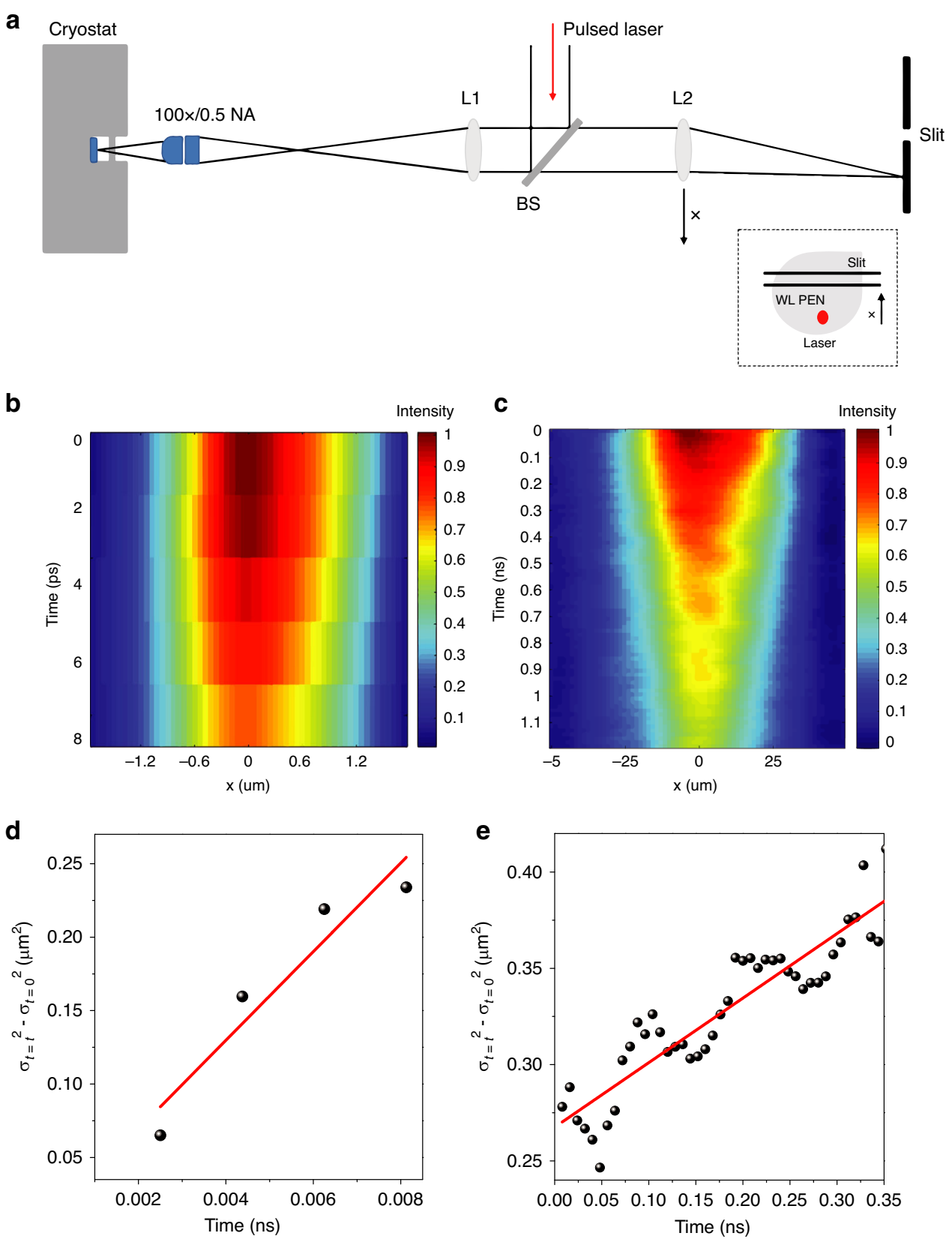

Fig. 4 Spatial-temporal mapping of $\mathbf{1 L}$ and WL PEN. a Schematic showing the experimental setup with a movable lens used for mapping. A pulsed 522-nm laser is focused onto $1 \mathrm{~L} / \mathrm{WL}$ placed on the copper cold finger in a cryostat using a beam splitter and a $\times 100 / 0.5-N A-l o n g$ working distance objective. Photoluminescence from the sample is imaged onto the entrance slit of the spectrometer using lenses L1 and L2. Lens L2 is translated to position the WL emission onto the entrance slit of the spectrometer. $\mathbf{b}, \mathbf{c}$ Spatial-temporal mapping for $1 \mathrm{~L}(\mathrm{WL})$, showing the diffusion in time and space. $\mathbf{d}$, e Time evolution of the mean square displacement of excitons in space for $1 \mathrm{~L}(\mathrm{WL})$. The diffusion coefficients obtained for $1 \mathrm{~L}$ and WL were $306.8 \pm 14.1$ and $3.3 \pm 1.1 \mathrm{~cm}^{2} / \mathrm{s}$, respectively, confirming the diffusion length observed from Fig. $3 \mathrm{~b}$

such as $2 \mathrm{~L}$ and bulk samples (both had J-aggregation). The $2 \mathrm{~L}$ pentacene sample also showed a sharp emission peak at $680 \mathrm{~nm}$, but the peak had a slightly larger width and a much lower PL intensity compared to 1L (Fig. S14). Additionally, bulk pentacene showed a broad PL emission, and the $680 \mathrm{~nm}$ emission peak was almost negligible (Fig.
S8) due to the significantly reduced coherence length caused by the disorder and interfacial states in the sample, similar to other bulk organic thin films ${ }^{33}$. In comparison, the 1L PEN sample showed a very strong and narrow linewidth PL emission at $680 \mathrm{~nm}$, which is a key characteristic of coherent emission. Thus, the lack of 
crystallinity, presence of more defect sites, inhomogeneous growth and high thickness compared to the $2 \mathrm{D}$ state lead to loss of coherent states in bulk thin film pentacene samples. Of course, our 1L samples were not perfect. There is still much room to improve the sample quality, which might give us a higher coherence length and a longer exciton diffusion length. Third, the quantum confinement of the system is beneficial to the speed of exciton transport since the confinement can increase the exciton oscillation strength ${ }^{27}$ (Supplementary information note 7$)$. Our $1 \mathrm{~L}$ pentacene samples ( $1.2 \mathrm{~nm}$ thick) have intrinsically high confinement at the 2D quantum limit. Moreover, the excitons in $1 \mathrm{~L}$ are highly anisotropic and can be aligned in a quasi-1D space along the $b$ axis, which further increases the confinement of exciton transport in $1 \mathrm{~L}$ pentacene. In summary, structural uniformity with a defect-free interface, highly confined excitons and excellent intermolecular coupling (large oscillator strengths) results in the supertransport of excitons in $1 \mathrm{~L}$ pentacene. On the other hand, the diffusion coefficient $\left(3.5 \mathrm{~cm}^{2} / \mathrm{s}\right.$; Fig. 3d) of the CT excitons in the WL sample was also much larger than that of other similar CT excitons reported recently ${ }^{24}$. Even though no coherence was observed in WL, the strong spatial confinement of the excitons $(\sim 0.6 \mathrm{~nm}$ thick) and the almost defect-free interfaces in WL samples led to long and fast propagation of long-lived CT excitons ${ }^{21}$. The high diffusion length obtained in WL PEN samples is critical for applications in high efficiency solar cells and charge separation ${ }^{52}$. In addition, waveguiding effects can be excluded, as the thicknesses of the $1 \mathrm{~L}$ and WL pentacene samples were $<1.5 \mathrm{~nm}$ and that of the h-BN layer underneath was only $\sim 4 \mathrm{~nm}$ (measured by AFM), thus making them insufficient for any light trapping and waveguiding, similar to previous reports $^{22}$.

\section{Discussion}

It is important to remark here that the observed diffusion corresponded to bright singlet excitons in our $1 \mathrm{~L}$ and WL pentacene samples and that the long-lived triplet excitons generated via the singlet fission in pentacene did not affect the diffusion measurements (Fig. 3). The reasons are twofold. First, the triplet states are non-radiative dark states ${ }^{53,54}$ and cannot be detected by the confocal photoluminescence setup we employed for our measurements $^{32}$. Second, the regeneration of emissive singlets via subsequent triplet fusion is not allowed in pentacene, and singlet fission in pentacene is expected to be exothermic and unidirectional ${ }^{55,56}$ because the relaxed triplet in pentacene has significantly less than half the energy of the singlet. This is in contrast to the situation in tetracene, where the near-degenerate singlet and triplet-pair energy levels permit both singlet fission and triplet fusion to occur.
It is also important to remark here that the observed supertransport of excitons in $1 \mathrm{~L}$ pentacene should be related to the unique properties of singlet fission in pentacene. First, singlet fission in pentacene, a fast nonradiative decay, occurs within a time scale of $\sim 80 \mathrm{fs}^{56}$. On the picosecond time scale, the singlet fission in pentacene thin films has a very small total yield of $\sim 2 \%{ }^{40}$. In our $1 \mathrm{~L}$ pentacene sample, both our pump-probe (Fig. 2d) and TRPL (Fig. 2c inset) measurements showed a monoexponential radiative decay of $\sim 4 \mathrm{ps,} \mathrm{which} \mathrm{indicates}$ minimal contribution of non-radiative singlet fission mechanisms in the 1-10-ps regime, consistent with a previous report ${ }^{40}$. Second, our $1 \mathrm{~L}$ pentacene sample at $77 \mathrm{~K}$ showed a much stronger PL intensity than our $2 \mathrm{~L}$ and bulk pentacene samples (Figs. 2b, S14 and S8), which suggests a highly reduced singlet fission rate in our $1 \mathrm{~L}$ sample. This could be attributed to the minimised disorder in our 1L samples and the impact of molecule packing on singlet fission in organic molecules ${ }^{57-60}$. Of course, further exploring the aggregation and layerdependent singlet fission in 2D pentacene samples would be a very interesting future topic.

\section{Superfast and angle-dependent transport of coherent excitons at room temperature}

Semiconductors with long-range and fast transport of excitons at room temperature are critical for future highspeed excitonic circuits and quantum computing devices ${ }^{13-15}$ that can operate at room temperature. Here, we also observed superfast and angle-dependent transport of excitons from $1 \mathrm{~L}$ pentacene samples at room temperature (Fig. 5). In our measurement system, the polarisation angle of the incident laser was fixed to be parallel to the diffusion mapping direction. The exciton diffusion lengths and effective diffusion coefficients along two axes $\left(b\right.$ and $a$, labelled as $0^{\circ}$ and $90^{\circ}$, respectively) of the pentacene unit cell were measured by rotating the sample with respect to the polarisation of the incident laser. The measured exciton diffusion length for the $1 \mathrm{~L}$ pentacene sample showed a maximum value of $0.93 \pm 0.09 \mu \mathrm{m}$ along the $b$ axis $\left(0^{\circ}\right)$ and a minimum value of $0.37 \pm 0.02 \mu \mathrm{m}$ along the $a$ axis $\left(90^{\circ}\right.$; Fig. $5 \mathrm{a}$, c). In contrast, the measured exciton diffusion length for the WL sample was not angle dependent, showing a constant value of $\sim 1.31 \pm$ $0.2 \mu \mathrm{m}$ (Fig. 5b, d). The exciton emission lifetime measured from the $1 \mathrm{~L}$ pentacene sample changed from $\sim 12.7 \mathrm{ps}$ at a polarisation angle of $0^{\circ}$ to $\sim 5.6 \mathrm{ps}$ at a polarisation angle of $90^{\circ}$ (Fig. 4e), whereas the lifetime measured from the WL pentacene sample remained largely unchanged $(\sim 2.8 \mathrm{~ns})$ with changing incident polarisation angle (Fig. 5e). The effective exciton diffusion coefficients were thus extracted and are shown in Fig. 5f. The $1 \mathrm{~L}$ pentacene sample showed clear anisotropic exciton transport, with a very high effective diffusion coefficient of $346.9 \pm 24.1 \mathrm{~cm}^{2} / \mathrm{s}$ along the $b$ axis and $95.3 \pm 10.2 \mathrm{~cm}^{2} / \mathrm{s}$ along the $a$ axis (Fig. $5 \mathrm{f}$ ), whereas 

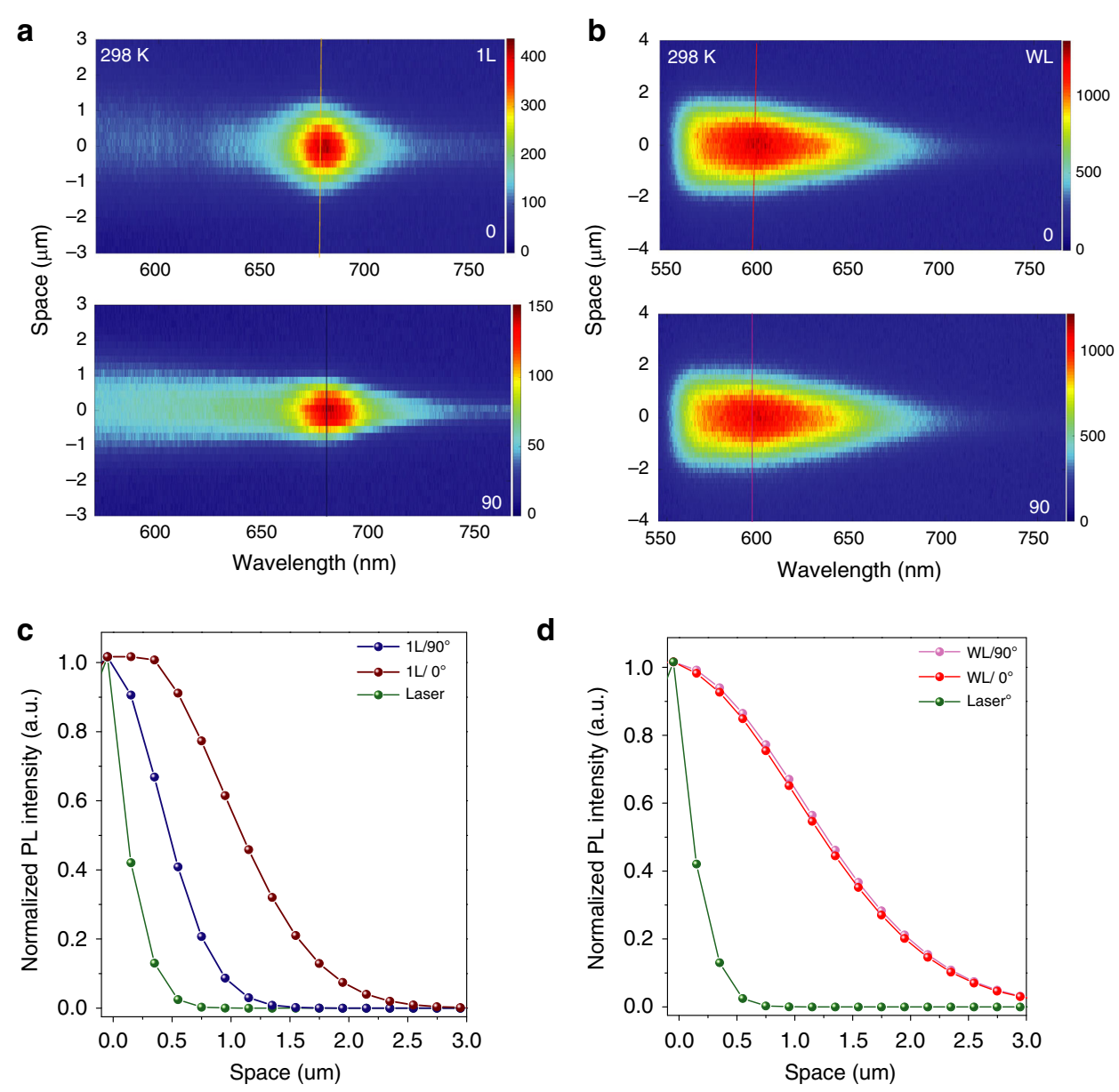

d
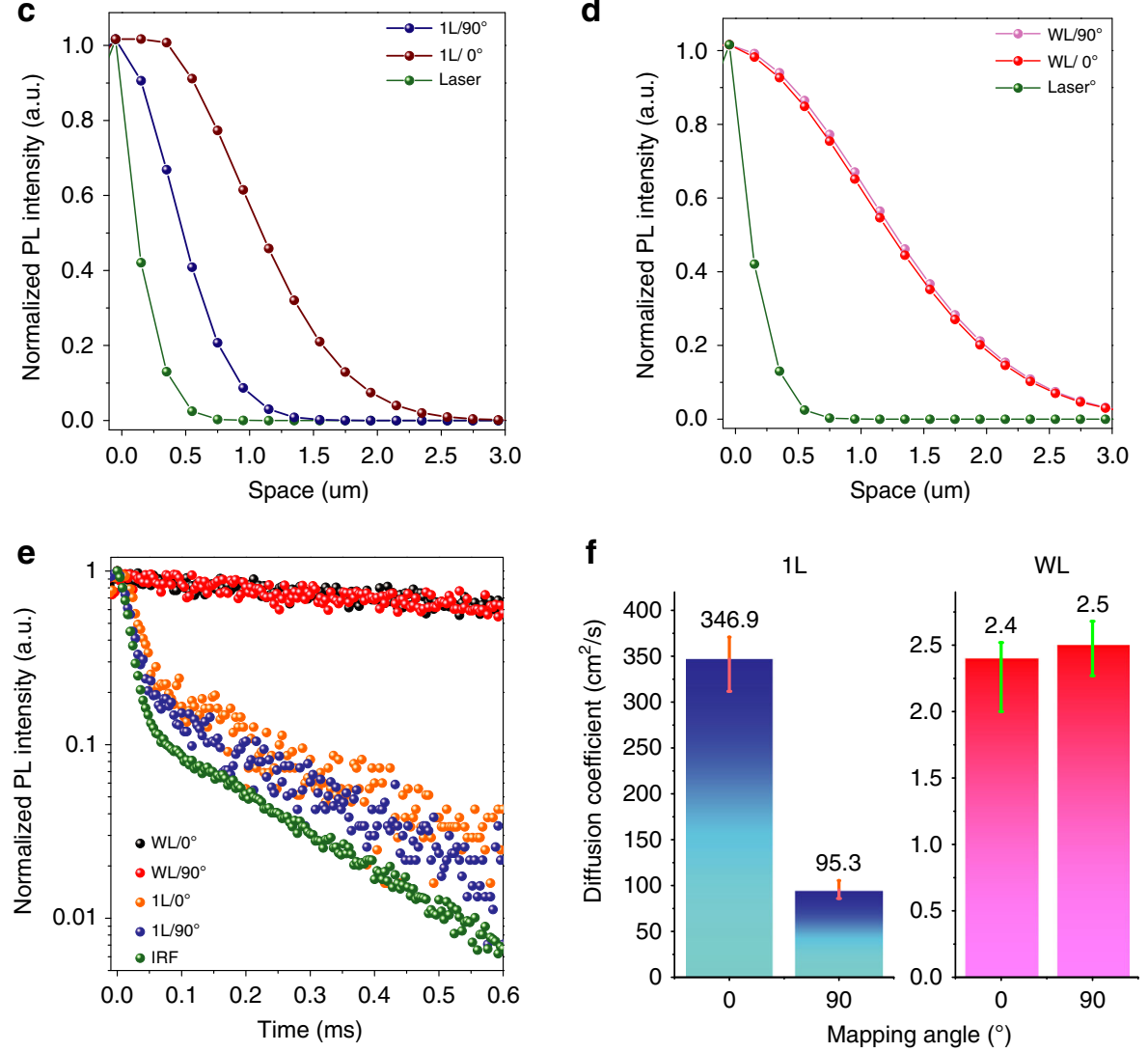

Fig. 5 Superfast and angle-dependent exciton transport at room temperature. $\mathbf{a}$, b Measured contour plots of PL intensity as a function of emission wavelength and space for $1 \mathrm{~L}(\mathbf{a})$ and $\mathrm{WL}(\mathbf{b})$ pentacene samples at room temperature, with spatial mapping direction parallel (top panel; marked as $0^{\circ}$ ) and perpendicular (bottom panel; marked as $90^{\circ}$ ) to the $b$ axis of the molecular lattices, as shown in Fig. S3a. In our measurement system, the mapping direction is parallel to the polarisation of the incident laser. The polarisation angles $0^{\circ}$ and $90^{\circ}$ have been marked according to the references set in the Fig. $2 \mathrm{f}$ inset. $\mathbf{c}$, $\mathbf{d}$ Spatial profile plots along the dotted lines in $\mathbf{a}, \mathbf{b}$ along two perpendicular mapping directions $\left(0^{\circ}\right.$ and $\left.90^{\circ}\right)$, showing the angle-dependent exciton transport in $1 \mathrm{~L}$ (c) and WL (d) pentacene samples. e Time-resolved PL emission (normalised) from WL and $1 \mathrm{~L}$ pentacene samples at room temperature. The black and red balls represent the decay curves measured from WL pentacene samples with incident polarisation angles of $0^{\circ}$ and $90^{\circ}$, respectively. Effective lifetimes of 2.8 and $2.8 \mathrm{~ns}$ were extracted from the black and red decay curves, respectively, by fitting with deconvolution of the instrument response function (IRF) (green dots). The orange and blue balls represent the decay curves measured from $1 \mathrm{~L}$ pentacene samples with incident polarisation angles of $0^{\circ}$ and $90^{\circ}$, respectively. The orange and blue decay curves were fitted with deconvolution of the IRF, giving effective short lifetime values of 12.7 and 5.6 ps, respectively. $\mathbf{f}$ Comparison plots of extracted diffusion coefficients measured from $1 \mathrm{~L}$ (left panel) and WL (right panel) samples under the designated mapping angles at room temperature 
the WL sample showed a consistent effective diffusion coefficient of $\sim 2.4 \pm 0.1 \mathrm{~cm}^{2} / \mathrm{s}$ along different axes of the sample due to its isotropic excitonic emission behaviour.

In conclusion, in high-quality atomically thin organic 2D semiconductors, we observed supertransport of excitons between coherent excitonic states with a large effective diffusion coefficient of $346.9 \mathrm{~cm}^{2} / \mathrm{s}$ at room temperature, which is $\sim 1$ to several orders of magnitude higher than the values reported for other organic quasi-1D and thin film molecular aggregates $^{19-21}$. We successfully identified molecular aggregation-sensitive PL emissions in atomically thin singlecrystal organic semiconductors with different types of aggregation ( $\mathrm{H}$ - and J-type) at the 2D quantum limit. The $1 \mathrm{~L}$ pentacene samples with J-type aggregation (FR exciton dominated) showed superradiant emission, which was experimentally confirmed by the temperature-dependent PL emission, highly enhanced radiative decay rate, significantly narrowed PL peak width and strongly directional in-plane emission. This can be attributed to the constructive dipole coupling in the J-aggregates in $1 \mathrm{~L}$ pentacene that forms an enhanced net optical dipole moment. The excitons in $1 \mathrm{~L}$ pentacene samples were determined to be delocalised over $\sim 135$ molecules. In addition, the supertransport of excitons in monolayer pentacene samples showed highly anisotropic behaviour. On the other hand, the WL pentacene samples with $\mathrm{H}$-type aggregation (CT exciton dominated) showed fast migration of isotropic $\mathrm{CT}$ excitons with a measured diffusion coefficient of $\sim 2.4 \mathrm{~cm}^{2} / \mathrm{s}$ at room temperature, a few times larger than that reported for other similar CT excitons ${ }^{24}$. Even though no coherence was observed in WL, the strong spatial confinement of the excitons $(\sim 0.6 \mathrm{~nm}$ thick) and the almost defect-free interfaces in WL samples lead to long and fast propagation of long-lived CT excitons at the 2D quantum limit ${ }^{21}$. Our results provide an important experimental demonstration of the long-range and superfast transport of excitons between coherent states at the 2D quantum limit, paving the way for promising applications in future highspeed excitonic circuits, quantum computing devices, fast OLEDs, and other optoelectronic devices.

\section{Materials and methods Material growth}

Few layer h-BN samples were prepared by mechanical cleavage method and placed onto a $\mathrm{SiO}_{2}$ layer which was thermally grown; $285 \mathrm{~nm}$ in thickness and deposited over a silicon substrate. The topology of the sample was examined by an optical microscope before physical vapour deposition (PVD) process. Pentacene samples (Chem Supply: P0030-1G) were then deposited over exfoliated h-BN which were placed in the centre of vacuum tube in furnace. The $\mathrm{h}-\mathrm{BN}$ samples were then placed around $15 \mathrm{~cm}$ downstream in the vacuum tube, and a pressure of $\sim 10^{-4}$ mbar was achieved in quartz tube using a molecular pump. The furnace temperature was increased to $130-150^{\circ} \mathrm{C}$ for $15-30$ mins to grow the pentacene samples. The furnace was then allowed to cool down naturally and vacuum was maintained. The number of layers were optimised by controlling the source temperature, growth time and substrate position. All the samples were characterised and the layer thickness was determined using atomic force microscope (AFM) measurements, in ambient room temperature conditions using a Bruker Multi-Mode III AFM instrument.

\section{Optical characterization}

Horiba LabRAM system combined with a confocal microscope was used to conduct PL measurements at both room temperature and $77 \mathrm{~K}$. A charge-coupled device (CCD) $\mathrm{Si}$ detector was used in conjunction with a 532-nm diodepumped solid-state (DPSS) laser which was used to excite the samples. For low temperature (above and at $77 \mathrm{~K}$ ) measurements, the samples were placed in a microscope-compatible chamber with a temperature controller (which used liquid nitrogen as the coolant). For polarisation dependent measurements the incident angle of polarisation of laser was controlled by a half-wave plate, and the angle of polarisation on the emission side $(\theta)$ was then determined using a polarizer which was angle-varied and placed in front of the detector. Time-resolved PL measurements were performed using a setup that incorporated $\mu$-PL spectroscopy and a timecorrelated single-photon counting (TCSPC) system. Pulsed laser which was linearly polarised (frequency doubled to $522 \mathrm{~nm}$, with a $300-\mathrm{fs}$ pulse width and a $20.8 \mathrm{-MHz}$ repetition rate) was channelled to a high numerical aperture $(\mathrm{NA}=0.7)$ objective (Nikon S Plan 603). The PL signal was then gathered by a grating spectrometer, thus either collecting the PL spectrum through a charge-coupled device (CCD; Princeton Instruments, PIXIS) or detecting the PL intensity decay using a Si single-photon avalanche diode (SPAD) and the TCSPC (PicoHarp 300) system. The double exponential decay from $1 \mathrm{~L}$ PEN is attributed to the biexponential decay in the instrument response function (IRF). The double exponential decay in the IRF is due to the delay in the response timings of the MPD SPAD ${ }^{\circledR}$ photon detector and the PicoHarp 300 coupled to it. A similar system response has been reported for an equivalent system $^{61}$. Similar biexponential decays are commonly reported for such systems ${ }^{62}$. Thus, we used deconvolution to extract a monoexponential decay curve for $1 \mathrm{~L}$, and all the lifetime data results were fitted after deconvolution with the IRF (Fig. 2c). This monoexponential decay was further confirmed by our TR pump-probe femtosecond resolution measurements, which also clearly demonstrated a monoexponential decay for $1 \mathrm{~L}$ PEN at $77 \mathrm{~K}$.

\section{Measurement of exciton diffusion lengths}

Exciton diffusion measurements were carried out using the same PIXIS CCD detector coupled with a $\times 100(\mathrm{NA}=1.49$, oil suspended) objective lens. A pulsed 522-nm laser was 
used for excitation with a beam diameter of $\sim 500 \mathrm{~nm}$ (confirmed by CCD imaging) and a collection time of $1 \mathrm{~s}$ per measurement. The diffusion mapping direction was well aligned with the polarisation of the excitation laser. The collected light was spectrally filtered to remove the pump laser wavelength. Spectral measurements were performed using a grating spectrometer (Acton, SpectraPro 2750). The focal plane of the sample was imaged using the zeroth order of the grating and the spectrometer $\mathrm{CCD}$, giving a spatial resolution of $\sim 200 \times 200 \mathrm{~nm}$ in space, corresponding to a pixel $(20 \mu \mathrm{m} \times 20 \mu \mathrm{m})$ on the CCD. The PL intensity at a particular excitonic emission energy was plotted as a function of the distance from the excitation centre ( $x=0$ in Figs. 3-4). The spatial extent of exciton diffusion (diffusion length $L_{D}$ ) was extracted by fitting the experimental data and laser profile with a 1D Gaussian diffusion model ${ }^{21,43}$. See Supplementary Information note 8 .

\section{Pump-probe transient reflectivity measurement}

Transient reflectivity measurements were performed with a home-built pump-probe setup. Degenerate, linearly polarised pump and probe pulses were produced by splitting $\sim 200 \mathrm{fs}$ pulses from a non-collinear optical parametric amplifier (Light Conversion Orpheus-2N) tuned to $680 \mathrm{~nm}$. A half-wave plate in the probe line rotated the polarisation to be orthogonal to that of the pump. The pump was delayed using a motorised delay stage to control the pump-probe timing. The pump and probe pulses were recombined to be nearly spatially overlapped and were focused onto the sample with a $25.4 \mathrm{~mm}$ focal length lens. Telescopes in the pump and probe lines were used to tune the divergence/waist size of the pump and probe beams. The focal spot size and fluence were $30 \mu \mathrm{m}$ and $90 \mu \mathrm{J} / \mathrm{cm}^{2}$ and were $15 \mu \mathrm{m}$ and $12 \mu \mathrm{J} / \mathrm{cm}^{2}$ for the pump and probe, respectively.

The probe pulses were spectrally resolved across a fast CMOS array detector (Andor Zyla) by an imaging spectrometer (Andor Kymera). The detector recorded spectra at $5.21 \mathrm{kHz}$, synced to the laser repetition rate of $20.83 \mathrm{kHz}$. A data analysis technique based on lock-in detection was used to isolate only the component of the probe modulated at the same frequency as the chopper in the pump beam path, which was also synced to the laser output. A polarizer was used to filter out the pump signal coupled with an iris. The residual pump signal still appeared minimally both as a background signal and as interference fringes between the pump and the probe. The interferometric pump contributions were removed using a Fourier filter with a width of $400 \mathrm{fs}$, and the static background was removed by subtracting the signal observed at negative pump-probe delays (i.e., the probe arriving before the pump). The data presented in Fig. 2 are the result of 11 and 5 repeats of a 15-ps scan of the pump- probe delay for $1 \mathrm{~L}$ and $\mathrm{WL}$, respectively, with a step size of $100 \mathrm{fs}$ and a 1-s dwell time at each step.

\section{Spatial-temporal mapping}

Spatial-temporal mapping was carried out on our samples at $77 \mathrm{~K}$ using a movable lens, focusing the PL signal onto the CCD detector with a movement range of $10 \mu \mathrm{m}$ from the central position on the sample (see the schematic in Fig. 4a). The temporal decay maps for $1 \mathrm{~L}$ and WL are consistent with the results shown in Fig. 3 of the main text. The data shown in Fig. S4b were smoothed in the post-processing by using one level of interpolation (image spline) between the recorded data from the system to extract the diffusion coefficient due to the limited number of data points in the regime.

\section{Acknowledgements}

We acknowledge funding support from ANU PhD student scholarship, China Scholarship Council, Australian Research Council (ARC; numbers DE140100805 and DP180103238) and ARC Centre of Excellence in Future Low-Energy

Electronics Technologies (project number CE170100039) and ARC Centre of Excellence in Quantum Computation and Communication Technology (project number CE170100012). We would also like to thank the facility support from Australian National Fabrication Facility ACT node, and from Professor Chennupati Jagadish and Professor Barry Luther-Davies at the Australian National University. We acknowledge the helpful discussions with Professor Haibo Ma and Xinran Wang from Nanjing University, China.

\section{Author details}

'Research School of Electrical, Energy and Materials Engineering, College of Engineering and Computer Science, The Australian National University, Canberra, ACT 2601, Australia. ${ }^{2}$ Optical Sciences Centre, Swinburne University of Technology, Hawthorn, VIC 3122, Australia. ${ }^{3}$ ARC Centre of Excellence for Future Low-Energy Electronics Technology, Australia. ${ }^{4}$ Centre for Quantum Computation and Communication Technology, Department of Quantum Science, Research School of Physics and Engineering, The Australian National University, Acton, ACT 2601, Australia. ${ }^{5}$ School of Mechatronical Engineering, Beijing Institute of Technology, Beijing 100081, China. ${ }^{6}$ Institute for Biomedical Materials and Devices (IBMD), Faculty of Science, University of Technology Sydney, Sydney, NSW 2007, Australia

\section{Author contributions}

Y.L. conceived and supervised the project; M.D., L.Z. and K.L. prepared pentacene samples; A.S. carried out all the optical measurements; A.S., Y.L. and R.H. analysed the data; A.S. took the AFM images; J.O.T., S.S., S.K.E., and J.A.D. helped with the TR pump-probe setup and measurements; H.T.N., D.M., T.V., P.K.L., Y.Z. and F.W. contributed to the setup for optical measurements; A.S. and Y.L. drafted the manuscript; and all authors contributed to the manuscript.

Conflict of interest

The authors declare that they have no conflict of interest.

Supplementary information is available for this paper at https://doi.org/ 10.1038/s41377-020-00347-y.

Received: 8 March 2020 Revised: 14 May 2020 Accepted: 9 June 2020 Published online: 06 July 2020

\footnotetext{
References

1. Shen, J. T. \& Fan, S. H. Coherent photon transport from spontaneous emission in one-dimensional waveguides. Opt. Lett. 30, 2001-2003 (2005).

2. Bradac, C. et al. Room-temperature spontaneous superradiance from single diamond nanocrystals. Nat. Commun. 8, 1205 (2017).
} 
3. Boulais, É. et al. Programmed coherent coupling in a synthetic DNA-based excitonic circuit. Nat. Mater. 17, 159-166 (2018).

4. Goban, A. et al. Superradiance for atoms trapped along a photonic crystal waveguide. Phys. Rev. Lett. 115, 063601 (2015)

5. Goldberg, D. et al. Exciton-lattice polaritons in multiple-quantum-well-based photonic crystals. Nat. Photon. 3, 662-666 (2009).

6. Lopez-Sanchez, O. et al. Ultrasensitive photodetectors based on monolayer MoS2. Nat. Nanotechnol. 8, 497-501 (2013).

7. Scheibner, M. et al. Superradiance of quantum dots. Nat. Phys. 3, 106-110 (2007).

8. Reimann, R. et al. Cavity-modified collective rayleigh scattering of two atoms. Phys. Rev. Lett. 114, 023601 (2015).

9. Chou, C. W. et al. Single-photon generation from stored excitation in an atomic ensemble. Phys. Rev. Lett. 92, 213601 (2004).

10. Wolke, M. et al. Cavity cooling below the recoil limit. Science 337, 75-78 (2012)

11. Bohnet, J. G. et al. A steady-state superradiant laser with less than one intracavity photon. Nature 484, 78-81 (2012).

12. Lunt, R. R. et al. Exciton diffusion lengths of organic semiconductor thin films measured by spectrally resolved photoluminescence quenching. J. Appl. Phys. 105, 053711 (2009).

13. Imamog-lu, A. et al. Quantum information processing using quantum dot spins and cavity QED. Phys. Rev. Lett. 83, 4204-4207 (1999).

14. Biolatti, E. et al. Quantum information processing with semiconductor macroatoms. Phys. Rev. Lett. 85, 5647-5650 (2000).

15. Solinas, P. et al. Holonomic quantum gates: a semiconductor-based implementation. Phys. Rev. A 67, 062315 (2003).

16. Singh-Rachford, T. N. \& Castellano, F. N. Photon upconversion based on sensitized triplet-triplet annihilation. Coord. Chem. Rev. 254, 2560-2573 (2010).

17. Dicke, R. H. Coherence in spontaneous radiation processes. Phys. Rev. 93, 99-110 (1954).

18. Abasto, D. F. et al. Exciton diffusion length in complex quantum systems: the effects of disorder and environmental fluctuations on symmetry-enhanced supertransfer. Philos. Trans. R. Soc. A 370, 3750-3770 (2012).

19. Wan, $Y$. et al. Direct imaging of exciton transport in tubular porphyrin aggregates by ultrafast microscopy. J. Am. Chem. Soc. 139, 7287-7293 (2017)

20. Caram, J. R. et al. Room-temperature micron-scale exciton migration in a stabilized emissive molecular aggregate. Nano Lett. 16, 6808-6815 (2016).

21. Clark, K. A., Krueger, E. L. \& Vanden Bout, D. A. Direct measurement of energy migration in supramolecular carbocyanine dye nanotubes. J. Phys. Chem. Lett. 5, 2274-2282 (2014).

22. Haedler, A. T. et al. Long-range energy transport in single supramolecular nanofibres at room temperature. Nature 523, 196-199 (2015).

23. Shaw, P. E., Ruseckas, A. \& Samuel, I. D. W. Exciton diffusion measurements in poly (3-hexylthiophene). Adv. Mater. 20, 3516-3520 (2008).

24. Zhu, T. et al. Highly mobile charge-transfer excitons in two-dimensional WS2/ tetracene heterostructures. Sci. Adv. 4, eaao3104 (2018).

25. Vörös, Z. et al. Long-distance diffusion of excitons in double quantum well structures. Phys. Rev. Lett. 94, 226401 (2005).

26. Sun, D. Z. et al. Observation of rapid exciton-exciton annihilation in monolayer molybdenum disulfide. Nano Lett. 14, 5625-5629 (2014).

27. Wu, S. D., Cheng, L. W. \& Wang, Q. Excitonic effects and related properties in semiconductor nanostructures: roles of size and dimensionality. Mater. Res. Express 4, 085017 (2017).

28. Lunt, R. R., Benziger, J. B. \& Forrest, S. R. Relationship between crystalline order and exciton diffusion length in molecular organic semiconductors. Adv. Mater. 22, 1233-1236 (2010).

29. High, A. A. et al. Control of exciton fluxes in an excitonic integrated circuit. Science 321, 229-231 (2008).

30. Grosso, G. et al. Excitonic switches operating at around 100 K. Nat. Photon. 3 577-580 (2009)

31. Baldo, M. \& Stojanović, V. Optical switching: excitonic interconnects. Nat. Photon. 3, 558-560 (2009)

32. Wan, Y. et al. Cooperative singlet and triplet exciton transport in tetracene crystals visualized by ultrafast microscopy. Nat. Chem. 7, 785-792 (2015).

33. Lim, S. H. et al. Exciton delocalization and superradiance in tetracene thin films and nanoaggregates. Phys. Rev. Lett. 92, 107402 (2004).
34. Zhang, Y. H. et al. Probing carrier transport and structure-property relationship of highly ordered organic semiconductors at the two-dimensional limit. Phys. Rev. Lett. 116, 016602 (2016).

35. Bardeen, C. J. Excitonic processes in molecular crystalline materials. MRS Bull. 38, 65-71 (2013).

36. Spano, F. C. \& Yamagata, H. Vibronic coupling in J-aggregates and beyond: a direct means of determining the exciton coherence length from the photoluminescence spectrum. J. Phys. Chem. B 115, 5133-5143 (2011).

37. Spano, F. C. The spectral signatures of frenkel polarons in $\mathrm{H}$ - and J-aggregates. Acc. Chem. Res. 43, 429-439 (2010).

38. Würthner, F., Kaiser, T. E. \& Saha-Möller, C. R. J-aggregates: from serendipitous discovery to supramolecular engineering of functional dye materials. Angew. Chem. Int. Ed. 50, 3376-3410 (2011).

39. Cong, K. K. et al. Dicke superradiance in solids. J. Opt. Soc. Am. B 33, C80-C101 (2016).

40. Marciniak, $H$. et al. Ultrafast singlet and triplet dynamics in microcrystalline pentacene films. Phys. Rev. B 79, 235318 (2009).

41. Montali, A. et al. Polarizing energy transfer in photoluminescent materials for display applications. Nature 392, 261-264 (1998).

42. Hestand, N. J. et al. Polarized absorption in crystalline pentacene: theory vs experiment. J. Phys. Chem. C 119, 22137-22147 (2015).

43. Kato, T. \& Kaneko, T. Transport dynamics of neutral excitons and trions in monolayer WS2. ACS Nano 10, 9687-9694 (2016).

44. Tokar, V. I. Non-Gaussian diffusion profiles caused by mobile impurity-vacancy pairs in the five frequency model of diffusion. arXiv 1801, 05285 (2018).

45. Ma, X. D. et al. Influences of exciton diffusion and exciton-exciton annihilation on photon emission statistics of carbon nanotubes. Phys. Rev. Lett. 115, 017401 (2015).

46. Mouri, S. et al. Nonlinear photoluminescence in atomically thin layered WSe2 arising from diffusion-assisted exciton-exciton annihilation. Phys. Rev. B 90 155449 (2014).

47. Akselrod, G. M. et al. Visualization of exciton transport in ordered and disordered molecular solids. Nat. Commun. 5, 3646 (2014).

48. Jailaubekov, A. E. et al. Hot charge-transfer excitons set the time limit for charge separation at donor/acceptor interfaces in organic photovoltaics. Nat Mater. 12, 66-73 (2013).

49. Saikin, S. K. et al. Photonics meets excitonics: natural and artificial molecular aggregates. Nanophotonics 2, 21-38 (2013).

50. Lloyd, S. \& Mohseni, M. Symmetry-enhanced supertransfer of delocalized quantum states. New J. Phys. 12, 075020 (2010)

51. Chuang, C. et al. Quantum diffusion on molecular tubes: Universal scaling of the 1D to 2D transition. Phys. Rev. Lett. 116, 196803 (2016).

52. Rao, A. et al. Exciton fission and charge generation via triplet excitons in pentacene/C60 bilayers. J. Am. Chem. Soc. 132, 12698-12703 (2010).

53. Smith, M. B. \& Michl, J. Singlet fission. Chem. Rev. 110, 6891-6936 (2010).

54. Piland, G. B. et al. Singlet fission: from coherences to kinetics. J. Phys. Chem. Lett. 5, 2312-2319 (2014).

55. Jundt, $C$. et al. Exciton dynamics in pentacene thin films studied by pumpprobe spectroscopy. Chem. Phys. Lett. 241, 84-88 (1995).

56. Wilson, M. W. B. et al. Singlet exciton fission in polycrystalline pentacene: from photophysics toward devices. Acc. Chem. Res. 46, 1330-1338 (2013).

57. Lukman, S. et al. Tuning the role of charge-transfer states in intramolecular singlet exciton fission through side-group engineering. Nat. Commun. 7, 13622 (2016).

58. Lukman, S. et al. Tuneable singlet exciton fission and triplet-triplet annihilation in an orthogonal pentacene dimer. Adv. Funct. Mater. 25, 5452-5461 (2015).

59. Roberts, S. T. et al. Efficient singlet fission discovered in a disordered acene film. J. Am. Chem. Soc. 134, 6388-6400 (2012).

60. Yost, S. R. et al. A transferable model for singlet-fission kinetics. Nat. Chem. 6 , 492-497 (2014).

61. Burgess, T. et al. Doping-enhanced radiative efficiency enables lasing in unpassivated GaAs nanowires. Nat. Commun. 7, 11927 (2016).

62. Bergmann, L. et al. Direct observation of intersystem crossing in a thermally activated delayed fluorescence copper complex in the solid state. Sci. Adv. 2, e1500889 (2016). 